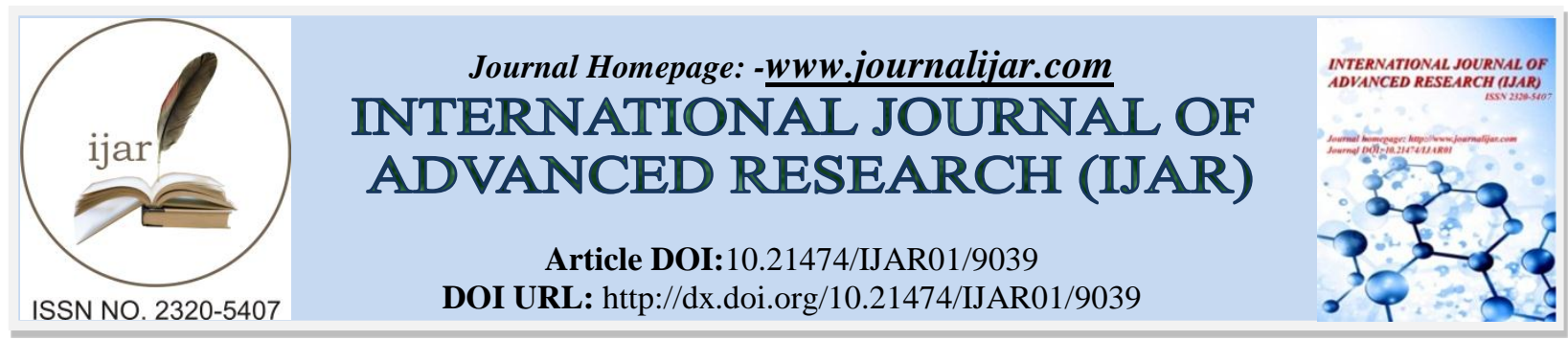

RESEARCH ARTICLE

\title{
PERCEIVING THE EFFECTIVENESS OF AN ENVIRONMENTAL MANAGEMENT SITUATION: CONTRIBUTION OF THE MODELING OF THE ENVIRONMENTAL BEHAVIOR OF THE ACTORS AS AN OBSERVATION INSTRUMENT.
}

Dr. Kabran Aristide Djane.

Université Peleforo Gon Coulibaly, Département De Sociologie.

\section{Manuscript Info}

Manuscript History

Received: 10 March 2019

Final Accepted: 12 April 2019

Published: May 2019

Key words:-

Modelling, Environmental behaviour,

Situation, Environmental management,

Environmental problem.

\begin{abstract}
Drawing on our work on modeling environmental behavior, in the context of solving environmental problems in schools, we present a discussion of the theoretical model resulting from the management of an environmental situation in primary school. The school is here a territory, a space of exchange and games of actors. This model, known as the "predictive funnel model", indicates that the management effectiveness factors of an environmental situation fall into two dimensions: an institutional dimension and an intrinsic dimension of the student.

Prenant ainsi appui sur nos travaux sur la modélisation du comportement environnemental, en regard à la résolution des problèmes d'environnement en milieu scolaire, nous présentons un modèle théorique issu de la gestion d'une situation d'environnement à l'école primaire. L'école étant ici un territoire, un espace d'échange et de jeux d'acteurs. Ce modèle baptisée "modèle entonnoir prédictif » indique que les facteurs d'efficacité de gestion d'une situation d'environnement relèvent de deux dimensions : une dimension institutionnelle et une dimension intrinsèque à l'élève.
\end{abstract}

Copy Right, IJAR, 2019,. All rights reserved.

\section{Introduction:-}

Les politiques environnementales trouvent leur efficacité dans le développement des normes qu'elles édictent, mais également dans le suivi des actions qu'elles mènent. Aussi la construction d'indicateurs en sciences sociales de l'environnement a-t-elle toujours permis le calibrage de l'observation sur le terrain. En effet, en Sciences Sociales, l'observation a toujours trouvé sa source dans l'opérationnalisation des concepts utilisés dans la recherche sociale. Il part ainsi, des dimensions des concepts, pour s'achever aux indicateurs. L'indicateur constitue ainsi la forme achevée et structurante de l'observation. Mais la construction de ces indicateurs constitue pour le chercheur, un exercice éprouvant, dénotant de l'expérience de ce dernier à la manipulation d'une grille de lecture appropriée à sa position théorique. Mais lorsqu'on passe à une phase pratique de la gouvernance de l'environnement, qui s'inscrit dans une gestion, la construction des indicateurs objectivement vérifiables devient un défi important pour le gestionnaire en environnement. De plus, la gestion ne devient efficace que par la réalisation des objectifs assignés dès le départ au gestionnaire. Aussi l'efficacité devient-elle une norme de gestion. Cette production a pour but de présenter une phénoménologie de la situation de gestion environnementale et son opérationnalisation à travers la modélisation du comportement environnemental. Elle construit alors à la présentation de la situation de gestion

Corresponding Author:-Dr. Kabran Aristide Djane.

Address:-Université Peleforo Gon Coulibaly, Département De Sociologie. 
environnementale, en s'appuyant sur le cas de la modélisation du comportement environnemental de l'élève. Enfin, elle discute les compétences comparées du sociologue de l'environnement et de l'ingénieur sociale de l'environnement dans le cadre d'une situation de gestion de l'environnement.

\section{La situation de gestion environnementale}

La situation de gestion environnementale découle lui-même du vocable de situation de gestion. Ainsi la gestion est la construction à partir d'un tableau de bord d'une conduite d'activités coordonnés au regard d'un objectif clairement défini. Cette approche se raffine avec la définition de Girin (1990) « une situation de gestion se présente lorsque des participants sont réunis et doivent accomplir, dans un temps déterminé, une action collective conduisant à un résultat soumis à un jugement externe ». Dans ce cas de figure, la prise en charge des problèmes d'environnement territorialisés conviendrait à être appeler les situations (territoriales) de gestion environnementale. Ainsi, la situation de gestion environnementale part d'une démarche qui prend les enjeux comportementaux des acteurs face à une question de problème d'environnement. Sauvé conçoit le problème environnemental comme étant une rupture d'équilibre environnemental. Cette rupture aboutit à une crise environnementale si l'on doit passer d'un équilibre $\mathrm{A}$ à un autre équilibre $\mathrm{B}$. Mais le dilemme apparait quand l'on doit répondre à la question du consensus dans un espace territoriale environnemental où la gestion est soit communautaire ou familiale. Dans tous les cas de figure, la nécessité de coercition dans le noyau représentationnel de l'environnement est constamment convoquée. De plus, la question de la situation de gestion environnementale fait également appel à la problématique du fait environnemental. Le fait environnemental reste le concept d'où découle l'action environnementale où le comportement environnemental prend également sa source. Nous, appuyant sur le concept définitionnel de comportement environnemental Kollmus et Agyeman (2002) résument le comportement environnemental en ces termes, « un comportement adopté par un individu qui décide, de façon consciente, de minimiser ses impacts négatifs sur les milieux naturel et construit » (Kollmus \& Agyeman, 2002, p. 240). Si ce comportement est adopté de façon permanente, l'éducation environnementale, qui la suscitée, se pérennise. Emmons (1997), cité par Musaferi (1998), effectue une nette distinction entre comportement environnemental et action environnementale. Pour cet auteur, l'action environnementale vise un but bien précis : elle doit être prévue et planifiée ; elle peut s'accomplir de façon individuelle ou communautaire et elle est volontaire et non automatique. A l'analyse, les définitions de Emmons et Kollmus (2002) sont parallèles et se complètent. Le comportement environnemental ne saurait se démarquer d'une action environnementale. Jensen et Schnack (1997) cité par Musaferi (1998) donnent néanmoins une distinction entre l'action environnementale directe et l'action environnementale indirecte. Elles sont dites directes si l'individu agit directement sur l'environnement pour améliorer le problème ; elles sont indirectes lorsque la personne en incite une autre à agir. C'est pourquoi, théoriser la situation de gestion environnementale devient un exercice complexe alliant une restructuration du système de jeux d'acteurs, confinés en stratégie individuelle ou collective.

Le dilemme de l'opérationnalisation de la situation de gestion environnementale : un dilemme de consensus représentationnel de l'environnement

Le dilemme de l'opérationnalisation de la situation de gestion environnementale devient une obligation de conception ou de matricialisation des représentations de l'environnement des acteurs en présence sur le fait environnemental ou auteurs du fait environnemental. Sauvé et Machabée (2000) indiquent sept niveaux représentationnels de l'environnement. Ces sept niveaux représentationnels ont chacun un enjeu comportemental de l'environnement. Ainsi, adopter un modèle de gestion de situation environnementale sans tenir compte de ces dimensions voue à l'échec, la démarche de gestion environnementale. Dans un autre temps, si une nécessité de conciliation des représentations et de consensus s'avère exigible, c'est la capacité d'extraction des variables servant à la conciliation représentationnelle de l'environnement qui confirme la qualité des experts en charge du développement de cette gestion. Sur cette question, un paradigme est conseillé par Robbotom et Hart (1993) et c'est en lui, que se réfugie cette production scientifique.

S'inspirant du paradigme interprétatif (Berthelot, 2007, p. 24), l'approche socioconstructiviste perçoit les sciences sociales de l'Environnement, comme un espace scientifique qui vise à permettre aux acteurs de développer une relation harmonieuse avec l'environnement, à la fois par l'émotion et la compréhension. La connaissance est vue comme subjective et tirée de l'expérience. La recherche est de type interprétatif, subjectif, constructiviste, qualitatif et éclairant ; elle est conduite par des experts externes selon un design préétabli, mais sensible aux besoins des acteurs. L'empathie et une relation harmonieuse avec l'environnement seront facilitées par l'organisation d'activités dans l'environnement. Le résultat attendu de l'intervention éducative est d'accroître la capacité à agir moralement et effectivement pour la préservation de l'environnement. Le programme et les guides d'activités fournissent au 
gestionnaire des indications et des suggestions d'expériences environnementales. Les acteurs sont perçus comme des apprenants actifs dont le cheminement suppose l'engagement, tant sur le plan émotif que cognitif, dans des expériences environnementales. Le rôle du gestionnaire de situation de l'environnement consiste à organiser des expériences signifiantes pour les élèves. L'évaluation s'attache à comprendre la manière dont les acteurs impliqués appréhendent la réalité et construisent leur propre éthique environnementale. Elle tente de les placer en situation de dialogue pour leur permettre de dégager un consensus sur leur vision des choses.

Van Matre (1990), qui privilégie un contact sensoriel avec l'environnement plutôt qu'une connaissance encyclopédique, est un représentant reconnu du courant interprétatif. De même, les travaux relatifs à l'éveil de la sensibilité et à une « pédagogie du sensible, de l'émotionnel et de l'imaginaire » en vue d'une réelle rencontre avec l'environnement et de l'établissement d'un lien «éco-logique » (Cottereau, 2000) ainsi que ceux qui s'intéressent au sens du lieu et à la relation au milieu (Pruneau, 1997); Pruneau, Chouinard et Gravel, (1998-1999) participent, selon Berthelot (2007, p. 25), de l'approche socioconstructiviste.

Le paradigme socioconstructiviste de Robbotom et Hart (1993) contribue à saisir le rapport société (culture) et la morphologie des attitudes adoptées par les individus avec leur environnement. Ils indiquent dans cet argumentaire que le lien avec l'environnement est basé sur du construit. A ce titre, il émane du socioculturel. L'extraction des variables servant à l'efficacité d'une situation de gestion environnementale émane donc de variable socioculturelle. Ainsi, l'exposition des variables résistantes et d'appropriation du fait environnemental sont donc déterminantes au succès d'une préoccupation environnementale.

En quoi, la modélisation enrichit-elle la situation de gestion environnementale ? : un questionnement épistémique et méthodologique au regard du modèle entonnoir prédictif

L'enjeu épistémique de cette production était de concilier par le biais du modèle entonnoir, les modèles purs (sciences de la Nature) et les modèles à déictiques (Sciences sociales) (Passeron, 2000). Par l'objet qu'il se propose de structurer à savoir le comportement environnemental de l'élève, le modèle entonnoir issu de notre modélisation clarifie la rencontre entre les sciences dites pures des Sciences de la Nature et les Sciences Sociales. Sauvé (2000) appelle cela, l'écosophie ou la philosophie de l'harmonisation des rapports de l'Homme à sa Nature, qui sous-tend la démarche également des sciences de gestion, avec pour option la gestion environnementale. La science Durkheimienne d'obédience causale rencontre donc, dans ce modèle entonnoir, les visions et méthodes wébériennes qui cherchent à mettre à jour des types idéaux de rapports c'est-à-dire des configurations de réponses (variables) inter-corrélées. Ce sont alors des variables vivantes (religion, beauté) croisées à des variables mortes (sexe) qui sont ici mis en exergue. La science Durkheimienne se relevant de variables mortes, tandis que celle wébérienne met à avant les variables vivantes. Le modèle entonnoir, concilie dans son développement, le modèle pur des Sciences de la Nature (Durkheimienne) et le modèle à déictiques (wébérienne). Il résout ainsi cette dichotomie épistémologique que relève Passeron (2000) entre les sciences de l'environnement dites naturelles et les sciences sociales qui incorporent les sciences de gestion.

\section{Le cas de l'environnement à l'école}

Le modèle entonnoir présente un plateau social sur lequel, il est bâti. En effet, les variables intrants que sont le sexe, la religion et la culture du beau obéissent toutes à un ancrage communautaire de la société ivoirienne. Les élèves impriment ainsi leur volonté de pratique environnementale tout en obéissant à une directive sociale. Même si les références à la beauté ont parfois un appui psychologique, elles indiquent que les enfants peuvent néanmoins, développer leur propre vision de la beauté. Cette subjectivité les met dans la plupart des cas à l'abri des influences sociales, mais qui dans la grande majorité, agit sur leurs décisions. Elle renforce alors les conclusions de Hart et Robbotom (1993) sur la nécessité que tout modèle sur le comportement environnemental et en Education Relative à l'Environnement doit impérativement, extraire son essence de la société sur laquelle, il repose ; dans notre cas de figure, sur la société ivoirienne où les us et coutumes obéissent à des rites initiatiques auxquels doivent se conformer les plus jeunes sous la surveillance des plus âgés. Le développement du modèle entonnoir conduit donc à la confirmation d'un apport essentiel au développement et à la compréhension des rapports sociaux que les jeunes élèves du primaire ont de/avec leur environnement. Le réalisme social ivoirien conforte ce modèle au regard des variables qui l'expliquent et du discours socioconstructiviste de Hart et Robbotom (1993).

Observer l'efficacité d'une situation de gestion environnementale recommande donc la construction par le gestionnaire d'une grille d'indicateurs matricialisant l'environnement qu'il gère. Cela suppose une connaissance parfaite des représentations, des comportements et des interconnexions entre les sources de représentations de 
l'environnement et les programmes de comportements environnementaux que les acteurs manipulent dans cette arène environnementale, qu'il gère.

Le cas du modèle entonnoir prédictif constitue ainsi un réalisme social de modélisation et d'observation d'une situation de gestion environnementale à l'école. Elle permet ainsi de faire ressortir les variables qui facilitent ou limitent l'acceptation du fait environnemental par les élèves en vue de recadrer les comportements environnementaux adéquat de ces acteurs quel que soit l'arène environnemental. L'efficacité de gestion environnementale qui s'en suit, n'est donc, toute autre que l'articulation judicieuse par le gestionnaire, de ses indicateurs relevés. Par ailleurs, elle soulève une discussion entre le statut du gestionnaire de la situation environnementale, les objectifs de sa fonction et la pertinence de ce statut à l'efficacité de la situation de gestion environnementale.

Ingénierie sociale de l'environnement, la situation de gestion environnementale et le sociologue de l'environnement : un complexe de réalité à un complexe d'expertises

Le dernier temps que convoque cette production est la complexification de la situation de gestion environnementale suivant le statut de celui qui l'applique dans l'espace donné. A ce titre, suivant les compétences de chaque acteur, chargé de la mise en place d'un programme alliant l'environnement à une pratique de matricialisation des indicateurs situés dans l'espace et l'environnement à construire ou à reconstruire, l'efficacité de cette situation de gestion s'accroit ou décline. Dans cette disposition, la situation de gestion environnementale implique une alliance d'expertise entre le sociologue de l'environnement et ceux des ingénieurs sociaux de l'environnement, tels que les architectes. A cette défense, Winkel et Al. (2009) indique que ces deux experts que sont le sociologue de l'environnement et l'architecte ou l'ingénieur social de l'environnement peuvent s'accorder dans une situation de gestion de l'environnement par ce que le psychologue de l'environnement appelle la sensibilité à l'environnement. Ainsi, même si leur outil d'investigation sociale de l'environnement diffère en vue de sa gestion, la sensibilité que leur prône le psychologue de l'environnement peut aider à combler ce gap. En effet, au-delà de la nécessité de la modélisation de l'espace à gérer comme le ferait un économiste ou un sociologue quantitativiste, l'espace à gérer conduit inexorablement à développer un partage des sensibilités en vue de s'accorder sur le maintien de l'état environnemental voulu. Sur cette disposition, Bozonnet (2012) explique que la sensibilité à l'environnement, même si elle repose sur les représentations sociales de l'environnement qu'ont les auteurs, requiert de convoquer leurs antécédents à l'environnement par observation aux types d'éducation environnementale qu'ils ont pu s'approprier dans leur cursus ou expérience de vie. Ainsi, même si la situation de gestion environnementale s'est vue prospérer dans les sciences sociales des organisations, elle a vite été entretenue par les sciences sociales de l'environnement qui lui ont trouvé une fonction assez pertinente celle d'expertiser les instruments de gouvernance de l'environnement.

\section{Conclusion:-}

Il s'est agi dans cette production de discuter le dilemme du gestionnaire des situations environnementales. Si ce dilemme se concentre dans une articulation judicieuse de faits environnementaux au profit des acteurs en présence, c'est plutôt la construction d'une grille d'indicateurs permettant une observation efficace de la réalité qui s'avère difficile pour le gestionnaire. Notre production a permis de redéfinir le concept de situation environnementale en articulant son observation empirique à partir des représentations des acteurs et de leurs logiques de comportements environnementaux qui en découlent; ainsi la mise en relation de ces sources de représentation à partir de leur noyau représentationnel conduit à une mixture matricielle que le gestionnaire doit savoir observer par la modélisation de ces sources. L'exemple du modèle entonnoir prédictif illustre bien cette attitude dans le cadre de la gestion des situations environnementales à l'école. Au terme de cette discussion, la sensibilité environnementale défendue par Bozonnet (2012) aide fondamentalement à concilier le dilemme des gestionnaires de la situation de l'environnement, qu'ils soient architecte, économiste ou sociologue de l'environnement.

\section{Références Bibliographiques :-}

1. Berthelot, M. (2007). Etude de la contribution du programme formation-information pour l'environnement à la perennisation de l'éducation relative à l'environnement dans l'enseignement primaire senegalais. Laval: Université Laval.

2. Boussard V. (2008), Sociologie de la gestion. Les faiseurs de performance, Belin, coll. « Perspectives sociologiques », 263p 
3. Bozonnet J-P. (2012). La sensibilité écologique. In Lewis N., et Al (Dir). Manuel de sociologie de l'environnement, Presses Universitaires de Laval, pp.147-162

4. Cottereau, D. (2000). Évaluer la mise en forme du lien éco-logique. Éducation relative à l'environnement Regards, Recherches, Réflexions, I, pp. 173-181.

5. Emmons, K. M. (1997). Perspective on environmental action : reflexion and revision through practical experience. The Journal of Environmental Education, XXIX(1), pp. 34-44.

6. Girin, J. (1990) L'analyse empirique des situations de gestion : éléments de théorie et de méthode, in A.-C. Martinet (dir.), Epistémologies et Sciences de Gestion, Paris : Economica, pp. 141-182.

7. Jensen, B., \& Schnack, K. (1997). The action cornpetence approach in environmental education. Environmental Education Research, III(2), pp. 163- 177.

8. Kollmus, A., \& Agyeman, J. (2002). Mind the gap: why do people act environmentally and what are the barriers to pro-environmental behaviour? Environmental Education Research, VIII(3), pp. 239-260.

9. Mermet, L. (1991). Dans quel sens pouvons-nous gérer l'environnement ? Annales des Mines/Gérer \& Comprendre, 22, pp. 68-81

10. Musaferi, J.-P. (1998). L'action environnementale dans la communauté. Les facteurs psychopédagogiques et culturels qui la motivent. Laval: Université Laval.

11. Passeron, J.-C. (2000). Le raisonnement sociologique. SFRS

12. Pruneau, D. (1997). Le sens du lieu : un concept prometteur en éducation relative à l'environnement. Vie pédagogique(105), pp. 8-9.

13. Pruneau, D., Chouinard, O., \& Gravel, H. (1998-1999). Le chaînon manquant : la compréhension de la relation personne-groupe social-environnement. Éducation relative à l'environnement - Regards, Recherches, Réflexions, pp. 209-215.

14. Sauvé, L., \& Machabée, L. (2000). La représentation : point focal de l'apprentissage. Éducation relative à l'environnement: Regards-Recherches-Réflexions, 2, 183-194.

15. Van Matre, S. (1990). Earth Education: A New Beginning. Warrenville, États-Unis: The Institute for Earth Education.

16. Winkel, G., Saegert, S., \& Evans, G. W. (2009). An ecological perspective on theory, methods, and analysis in environmental psychology: Advances and challenges. Journal of Environmental Psychology, 29(3), 318-328. 